\title{
Incidencia de las metodologías de enseñanza en las carreras de ciencias administrativas ofertadas por las universidades públicas del DMQ.
}

Incidence of teaching methodologies in the careers of administrative sciences offered by the public universities of the DMQ.

Giovanni Herrera Enríquez. ${ }^{1}$, Sergio Castillo Páez. ${ }^{2}$, Danny Zambrano Vera. ${ }^{3}$, Maybelline Jacqueline Herrera Sánchez. ${ }^{4}$, \& César Iván Casanova Villalba. ${ }^{5}$.

DOI: https://doi.org/10.33262/visionariodigital.v5i1.1526

\begin{abstract}
.
Introduction: The diffusion of knowledge is part of the evolution and human development, in this space the higher education plays a fundamental role in the search, diffusion and construction of the knowledge. Several methods of teaching have been developed to allow the students to assimilate the learning. Objective: To determine the effectiveness of the various teaching methods given by teachers to students at the undergraduate level, focusing the student's perspective on the methods applied by their teachers and the conception of learning. Methodology: This research is of a theoretical type based on documents, with the use of tools such as the application of surveys,
\end{abstract}

${ }^{1}$ Universidad de las Fuerzas Armadas ESPE. Sangolquí - Ecuador, E-mail: gpherrera@espe.edu.ec. ORCID: https://orcid.org/0000-0002-2835-4586

${ }^{2}$ Universidad de las Fuerzas Armadas ESPE. Sangolquí - Ecuador, E-mail: sacastillo@espe.edu.ec. ORCID: https://orcid.org/0000-0002-5402-2462

${ }^{3}$ Universidad de las Fuerzas Armadas ESPE. Sangolquí - Ecuador, E-mail: dizambrano@espe.edu.ec. ORCID: https://orcid.org/0000-0002-5920-0790

${ }^{4}$ Universidad Técnica Luis Vargas Torres de Esmeraldas. Santo Domingo - Ecuador. E-mail: maybelline.herrera.sanchez@utelvt.edu.ec. ORCID: https://orcid.org/0000-0001-6840-3891

${ }^{5}$ Universidad Técnica Luis Vargas Torres de Esmeraldas. Santo Domingo - Ecuador. E-mail: cesar.casanova.villalba@utelvt.edu.ec ORCID: https://orcid.org/0000-0001-6486-1334 
giving an exploratory descriptive result, applying an analytical deductive method. Results: the most applied methods by the teachers are master conferences and problembased learning, however, the case study and simulation methods are qualified by the students as the ones that provide better results for the learning capture. Conclusion: The results of the research were analyzed for the final year students of the Business Administration, Accounting and Auditing careers at the Central University of Ecuador and the University of the Armed Forces ESPE, and there are two important conclusions: the first is that the students do not have a correct interpretation of each concept of the different methods and therefore do not understand the difference between their application; the second is that the teachers do not look for new ways to reach the students and they are pigeonholed into the typical methods, for this reason there is no circumstantial difference in knowledge.

Keywords: Teaching methodologies, teaching methods, student perspectives, conception of learning, Public Universities of Quito.

\section{Resumen.}

Introducción: La difusión del conocimiento forma parte de la evolución y el desarrollo humano, en este espacio la educación superior juega un papel primordial en la búsqueda, difusión y construcción del conocimiento. se han desarrollado varios métodos de enseñanza para permitir a los estudiantes asimilen el aprendizaje. Objetivo: Determinar la efectividad de los diversos métodos de enseñanza impartidos por los docentes a los estudiantes en nivel de pregrado, focalizando la perspectiva del estudiante sobre los métodos aplicados por sus docentes y la concepción del aprendizaje. Metodología: esta investigación es de tipo teórica basada en documentos, con la utilización de herramientas como la aplicación de encuestas, dando un resultado descriptivo exploratorio, aplicando un método deductivo analítico. Resultados: los métodos más aplicados por los docentes son conferencias magistrales y el aprendizaje basado en problemas, sin embargo, los métodos de estudios de caso y simulaciones son calificados por los estudiantes como los que brindan mejores resultados para la captación del aprendizaje. Conclusión: Se analizó los resultados de la investigación a los estudiantes del último año de las carreras de Administración de Empresas, Contabilidad y Auditoría de la Universidad Central del Ecuador y de la Universidad de las Fuerzas Armadas ESPE, y se tienen dos conclusiones de trascendencia, la primera que los estudiantes no tienen una correcta interpretación de cada concepto de los diferentes métodos por ende no conciben la diferencia entre su aplicación, la segunda es que los docentes no buscan nuevas formas de llegar a los estudiantes y se encasillan en los métodos típicos, por tal motivo no existe una diferencia de circunstancial en conocimientos. 
Palabras claves: Metodologías de enseñanza, Métodos de enseñanza, perspectivas estudiantiles, concepción de aprendizaje, Universidades Públicas de Quito.

\section{Introducción.}

La responsabilidad permanente del profesor abarca la enseñanza-aprendizaje, la investigación y la vinculación con la sociedad, las cuales están interrelacionadas, no obstante, la presente investigación se concentró en la enseñanza, donde el docente genera curiosidad, expectativas y compromisos con sus estudiantes y con la humanidad. Así, el papel del profesor no está limitado a que conozca el contenido de una asignatura para enseñarla, puesto que la transferencia de conocimientos específicos no se debe considerar como suficiente tarea de la docencia. Corroborando con esa concepción sobre la docencia, Delors (1996), expone que la profesión de un docente no es simplemente el dictar una clase, si no que el objetivo de un docente es el transmitir conocimientos para que el estudiante pueda solucionar problemáticas de su profesión.

De acuerdo a Cullen (1997), el educador debe impartir una enseñanza trascendente la cual, tomando como analogía, aquella persona que después de permanecer en la caverna sale de ella y contempla la luz y la belleza del mundo, vuelve a la caverna para ayudar a otros a salir de la obscuridad, lo que semeja a que los docentes deben compartir sus conocimientos y los estudiantes, los cuales deberán utilizar estas enseñanzas con fines profesionalizantes. El principal desafío colocado al profesor universitario tal vez sea el de dar respuesta adecuada a las reformas ocurridas en la educación superior y orientar su docencia para el proceso de enseñanza-aprendizaje. Andrade (2004) comenta que la enseñanza aprendizaje debe estar en concordancia con el modelo de profesionales que se desea formar, bajo un pensamiento de formación de la academia con la metodología pedagógica acorde.

Al observar la importancia de las prácticas de enseñanza, que aproximen a los discentes a la realidad del mercado y de la profesión, se puede formular la siguiente pregunta: ¿cómo deben ser las prácticas pedagógicas en el área de Administración y sus impactos en el desempeño profesional en la visión de los graduados de pregrado en esa área? Para sustentar este cuestionamiento Aktouf (2005) afirma que la enseñanza de la ciencia de administración debe cambiar de ser un método de reproducción y dejar de lado el conservadurismo y/o conformismo, o sea, abrirse hacia la creatividad. Además, es relevante que las IES paren de "formar clones" y que los profesores se comprometan con la creatividad y el cambio.

Una vez que la excelencia de la enseñanza y del aprendizaje en la educación superior es cuestionada por varios sectores de la sociedad, la competencia pedagógica presentada por los profesores se torna un aspecto importante para la calidad en los programas. Esta relación de consecuencia es también sustentada por Lindberg-Sand y Sonesson (2008). Casanova (2018) detalla que la calidad debe mantenerse tanto en el sector público como privado, en el caso de 
educación se deberían tener estándares más altos lo cual también es el pensamiento de Herrera (2018).

Rosselló (2005) se refiere a la didáctica general que, a partir de la década de los ochenta, presenta las siguientes particularidades:

a. Contexto científico y epistemológico. Surgen nuevas formas de concebir y construir el conocimiento científico por lo que es cuestionado el enfoque positivista como la única vía para crear conocimiento científico.

b. La postmodernidad y la sociedad del conocimiento favorecen el surgimiento de nuevas maneras de conocer la realidad, de relacionarnos, de enseñar y de aprender.

c. Debido a los cambios que se mencionaron anteriormente, las profesiones requieren que la función del docente colabore con una conducción que aproxime el estudiante al mundo del trabajo (formación ocupacional, formación profesional, la enseñanzaaprendizaje con TIC en el ámbito universitario) (p. 138).

Los diversos métodos de enseñanza son herramientas o canales disponibles para ser utilizados por el profesor para construir una relación con el estudiante, dirigiendo el aprendizaje. La utilización de un método especifico, de manera correcta, en el momento correcto, consigue facilitar al estudiante la asimilación, procesamiento, crítica, comprensión e incorporación de los conocimientos de un determinado estudio que esté siendo trabajado.

Es importante considerar que la educación no cambia con la introducción de medios y productos tecnológicos, porque estos son acoplados dentro de la pedagogía y didáctica, articulándolos para el alcance de objetivos de la educación. Los estudiantes, durante su permanencia en el curso, experimentan varias formas de enseñanza, como efecto, son ellos los que experimentan y son capaces de discernir la calidad, la pertinencia y la utilidad del conocimiento colocado a su disposición. Los estudiantes están calificados para expresar su satisfacción o insatisfacción con la experiencia educativa. Ellos tienen derecho a expresar sus opiniones, en cualquier caso, y nadie más puede reportar la medida en que esa experiencia fue útil, productiva, informativa, satisfactoria.

Se debe destacar que los profesores tienen un control limitado sobre varios factores de aprendizaje (Carreño, 2012), debido a que existen otros elementos que también tienen influencia sobre el aprendizaje del estudiante, como su preparación emocional para aprender, los conocimientos previos sobre el contenido del curso, las habilidades de aprendizaje, el tiempo que el estudiante dedica en su aprendizaje, y otros elementos relevantes de cada alumno. Se puede argumentar que lo que aprende el estudiante no siempre es la consecuencia de una responsabilidad compartida entre el profesor y el estudiante. Además, existen muchos estudiantes capaces de aprender a pesar del profesor, mientras que otros fracasan a pesar de todos los esfuerzos de un profesor experto en la materia. 
Para efectos de la presente investigación se analizarán los principales métodos de enseñanza como son conferencias magistrales, estudio de caso, el de discusión en grupo, el aprendizaje basado en problemas, la investigación, simulación, seminario y juego de roles (Sáez, 2018), métodos que son aceptados y utilizados dentro de la comunidad académica y considerados como los más utilizados y con mejores resultados de aprendizaje.

En este estudio se identifica como problema de investigación los siguientes cuestionamientos ¿cuáles son las metodologías de enseñanza declaradas? y si ¿ellas son aplicadas para consolidar el aprendizaje de los estudiantes?, con lo que se pretende cumplir el objetivo de investigar la calidad de las actividades de enseñanza, definida como la consolidación del aprendizaje en base de la aplicación de distintos métodos impartidos a los estudiantes del último año de las Carreras de Administración de Empresas y de Contabilidad y Auditoría, ofrecidas por la Universidad de Las Fuerzas Armadas ESPE y por la Universidad Central del Ecuador - UCE, en 2018.

\section{Metodología.}

Como metodología inicial se tomó en consideración los criterios de Blaich y Wise (2011) que definen el uso de la evidencia del aprendizaje de los estudiantes como cinco pasos que ayudan a las instituciones a identificar procesos efectivos de evaluación y el uso de estos datos. Estos pasos incluyen la auditoría de datos; Involucrar a la facultad, el personal y los estudiantes para conversar y diseñar un plan antes de recopilar datos de evaluación en preparación para la reflexión; Asegurar recursos (dinero, espacio, tiempo, etc.) para fomentar la colaboración interinstitucional regular para el diálogo sobre los datos; Centrar los esfuerzos de evaluación en uno o dos resultados basados en la evidencia; Y alentar la participación de los estudiantes con los datos de la evaluación para ayudar en la interpretación de los resultados.

Realizada la planificación o estructura del proyecto según los pasos anteriores, para la recolección de datos se utilizaron encuestas y entrevista por medio de cuestionarios, por lo que se estructuró el instrumento de investigación que fue aplicado a los estudiantes del último año de las carreras de Administración de Empresas, Contabilidad y Auditoría de la Universidad Central del Ecuador y de la Universidad de las Fuerzas Armadas ESPE.

\section{Variables estudiadas}

En función de acceso a los datos, la variable operativa seleccionada fue rendimiento y satisfacción de los estudiantes, la cual es una variable compuesta, que se especifica a continuación:

Variables relacionadas con el rendimiento y satisfacción de los estudiantes:

- Condiciones de docencia. 
- Calificación subjetiva (merecida para el estudiante).

Con la finalidad de obtener los datos bases para el estudio de las dos variables, se realizaron dos cuestionarios, uno para cada variable estudiada, pero se aplicaron a la misma muestra representativa de estudiantes. El primer cuestionario cubre los criterios de que el método es utilizado de acuerdo a su concepción, su facilidad en comparación con otros métodos y por último el grado de contribución a las calificaciones respondiendo a la variable calificación subjetiva del estudiante; en el segundo cuestionario se aborda conceptos puntuales de cada método con el énfasis de analizar la aplicabilidad por parte del cuerpo docente correspondiente a la variable de condiciones de docencia.

\section{Recolección de información}

Fueron diseñados varios instrumentos para la recolección de información sobre las variables señaladas, tanto cuestionarios como protocolos de entrevista, pero, el enfoque de la investigación se centró en los resultados de los cuestionarios, los que fueron elaborados por el equipo de investigación para obtener información sobre los métodos de enseñanza, respondiendo a una selección exhaustiva de ítems representativos de los dominios de contenido objeto de análisis. La variable "Condiciones de docencia" se configura con distintas dimensiones: características del aula, metodología didáctica, materiales y recursos utilizados, implicación del alumno en el proceso docente, estrategia evaluativa, prácticas y valoración global. La medida de estas variables se realiza a partir de un cuestionario propio de $\mathrm{N}$ ítems, en formato tipo Likert con cinco categorías de respuesta.

\section{Muestra}

Dado el interés en analizar escenarios de enseñanza en el desarrollo curricular, la muestra fue conformada por estudiantes de los últimos niveles de las carreras de Administración de empresas, Contabilidad y Auditoría de ofrecidos en las universidades de análisis, siendo un total de 185 estudiantes encuestados. En esta oportunidad se utilizó un muestreo no probabilístico para lo cual se requirió construir conglomerados, los cuales fueron compuesto por grupos de estudiantes del último año de la carrera, se eligió un grupo y a cada individuo se les aplicó los cuestionarios.

\section{Análisis de consistencia del instrumento}

Para cada sección del instrumento, correspondiente a cada uno de los métodos estudiados, se analiza la consistencia interna de las respuestas de aquellos estudiantes que declaran que el método fue efectivamente utilizado. Para esto, se calcula el alfa de Cronbach en cada subgrupo de ítems, y se observa que este estimador presenta valores aceptables (cercanos o superiores a 0.70 ) en todos los casos.

Como una medida global de consistencia, este análisis también se aplica para todos los estudiantes que declaran haber utilizado todos los métodos, los cuales son 114 de la muestra 
de 185 encuestados (61.6\%), los que declaran que han utilizado todos los métodos. En este caso, el alfa de Cronbach es también alto, indicando que el instrumento es consistente de forma general. Además, es importante indicar que, de acuerdo a este análisis, ninguno de los ítems utilizados debió ser eliminado, lo que corrobora la consistencia del instrumento.

\section{Resultados.}

\section{Análisis estadístico}

Los estudiantes son evidencias racionales y fuente confiable (Arreola, 1995; Braskamp y Ory, 1994; Pratt, 1997). Los estudiantes están calificados para expresar su satisfacción o insatisfacción con la experiencia educativa. Ellos tienen derecho a expresar sus opiniones, en cualquier caso, y nadie más puede reportar la medida en que la experiencia fue útil, productiva, informativa, satisfactoria. Bajo este enfoque el levantamiento de datos se realizó en la Carreras de Administración de Empresas, Contabilidad y Auditoría de la Universidad de las Fuerzas Armadas ESPE y en las de la Universidad Central del Ecuador, por lo que el análisis fue, inicialmente, aplicado a cada universidad y a seguir se compararon los resultados obtenidos entre las universidades.

\section{Análisis de la utilización de los métodos de enseñanza aprendizaje}

Díaz y Hernández (1998) enfatizan que los métodos de enseñanza se enfocan en factores como el diseño y el empleo de objetivos de enseñanza, aporte que se trata de demostrar con la utilización y calificación de la satisfacción por parte de los estudiantes, se presenta los resultados de la aplicación de los cuestionarios para medir en primera instancia la frecuencia de uso para cada método utilizado por los diferentes docentes, en las carreras de estudio, los ítems están clasificados en la frecuencia de uso de cada método, así como la relación entre la teoría del método y la aplicación áulica, y como estos dos factores se afectan al aprendizaje que se mide con las calificaciones obtenidas por los estudiantes.

\section{Frecuencia de uso para cada método}

De acuerdo con la frecuencia de uso para cada método, se puede comprobar que todos los métodos tienen un alto porcentaje de uso (superior al $80 \%$ en todos los casos). Los métodos más utilizados son las conferencias magistrales (CM), estudio de caso (EC), el de discusión en grupo (GP) el aprendizaje basado en problemas (ABP), la investigación (INV) y la Simulación (SIM). Los métodos menos frecuentes corresponden al seminario (SEM) y al juego de roles (MJR).

\section{Concordancia entre la fundamentación teórica del método y su aplicación en el aula.}

La mayoría de los métodos utilizados en el aula concuerdan en un grado alto o muy alto con su fundamentación y características teóricas, es decir que si se ejecutan según las características del método. Sin embargo, esto no sucede con el uso de la Conferencia 
Magistral, seguido por el Estudio de Casos, en esos dos métodos se observa una valoración neutral alta. Los métodos con mayor concordancia en este sentido corresponden a Juego de Roles y Simulación, siendo el primero de estos uno de los métodos con la menor frecuencia de uso. Los resultados también nos arrojan que no hay claridad en la percepción de los estudiantes sobre las características teóricas del método para poder compararlas con su aplicación en sala de clase.

\section{Relación entre el método utilizado y la facilidad de aprendizaje.}

Si se analiza como contribuye el método utilizado en clase con la facilidad de aprendizaje por parte del estudiante, se puede observar que todos los métodos presentan, en la mayoría de los casos, una efectividad en grado positivo o neutro. No obstante, al parecer de los encuestados los métodos más efectivos corresponden al Estudio de Caso, la Simulación y el Juego de Roles. Entre los métodos menos valorados positivamente se encuentran la el Seminario, el Aprendizaje Basado en Problemas y la Investigación. En lo que se relaciona a los métodos Aprendizaje Basado en Problemas y de Investigación, la dificultad del estudiante en el aprendizaje puede demostrar una preparación académica deficitaria que no le permite visualizar claramente la solución de problemas y ejercer la lógica de la metodología de investigación. De los resultados se desprende que la enseñanza practicada por los docentes no alcanza transmitir suficiente preparación a los estudiantes de manera que ellos puedan asimilar mejor los métodos Aprendizaje Basado en Problemas y el de Investigación, lo que dificulta su aprendizaje.

\section{Incidencia del método utilizado en la calificación obtenida.}

En este ítem se analiza la percepción del estudiante en cuanto al grado de incidencia que tiene el método utilizado en clase respecto a su calificación (nota) obtenida. De manera general, se verifica que se reporta una alta relación de todos los métodos con la calificación obtenida cuando la valoración es aceptable (entre 30\% y 46\%). Este resultado parecer ser más evidente para los casos de la Conferencia Magistral y el Seminario En caso de una valoración muy alta, esta se encuentra entre $24 \%$ y $37 \%$. Por otra parte, el uso del método de Aprendizaje Basado en Problemas presenta una mayor incidencia neutra respecto a calificación obtenida.

En esos resultados obtenidos de los encuestados de la UCE, también se puede interpretar que los estudiantes podrían esperar una mejor calificación caso los métodos fuesen aplicados, puesto que Seminario, Juego de Roles y Aprendizaje Basado en Problemas fueron identificados como poco utilizados en las carreras estudiadas.

\section{Resultados específicos para cada método de enseñanza}

Para el análisis de la segunda variable, la cual se relaciona con los resultados de cada método según los atributos que contribuyen a la enseñanza, se presentan los resultados objetivos recabados de los estudiantes encuestados. Como se mencionó en la metodología se realizaron 
dos encuestas las cuales los ítems unos, dos y tres fueron analizados en el apartado anterior, por lo cual para los siguientes ítems se utilizarán las siglas del método analizado y las numeraciones subsiguientes.

\section{Análisis de resultados específicos para: Conferencias Magistrales}

Se analizaron los siguientes ítems específicos:

- $\quad$ CM4: Experiencia y conocimiento del docente

- CM5: Participación de los estudiantes

\section{- $\quad$ CM6: Lenguaje claro y accesible para la audiencia}

Rodríguez (2011) señala que el método conferencias magistrales pese a tener más de ocho siglos es uno de los métodos más utilizados pero que se lo debe empatar o combinar con otros métodos para que tenga un mayor impacto. La tendencia general de respuesta en todos los casos es hacia valores altos de la escala. Adicionalmente, en todos los ítems, aproximadamente las dos terceras partes de los encuestados valoran de forma aceptable o muy satisfactorio el conocimiento y experiencia del docente, la participación de los estudiantes y la claridad del lenguaje utilizado en este método. Por la valoración obtenida se desprende que la percepción de los estudiantes es de que el docente practica satisfactoriamente método Conferencia Magistral en su tarea de enseñanza.

\section{Análisis de resultados específicos para: Estudio de Caso}

Se analizaron los siguientes ítems específicos:

- $\quad$ EC4: Explicación metodológica del docente

- $\quad$ EC5: Fomento del pensamiento creativo

- $\quad$ EC6: Evaluación basada en desempeño

La tendencia general de respuesta en todos los casos es hacia valores altos de la escala. Adicionalmente, en todos los ítems, aproximadamente las dos terceras partes de los encuestados valoran de forma aceptable o muy satisfactorio cada uno de ellos, siendo más clara esta tendencia para los aspectos relacionados con el fomento del pensamiento creativo y la evaluación basada en el desempeño.

\section{Análisis de resultados específicos para: Simulación}

Se analizaron los siguientes ítems específicos:

- $\quad$ SIM4: Logro de objetivos del método 
- $\quad$ SIM5: Evaluación consensuada del problema de estudio

- $\quad$ SIM6: Ejercicios pertinentes a la realidad profesional

La tendencia general de respuesta en todos los casos es hacia valores altos de la escala, siendo un poco más leve este comportamiento para el logro de objetivos del método.

Álvarez (2001) considera que la formación de los estudiantes debe ejecutarse con actividades que den una visión integradora para la preparación de actividades laborales. Este método pone a la enseñanza en una posición de aproximación con la realidad que es valorizada por más de dos terceras partes de los estudiantes. Sin embargo, casi una tercera parte de los estudiantes encuestados no valoriza la relación de los ítems con la aplicación del método. Ello significa que la enseñanza del docente en el desarrollo del método no llega a todos sus estudiantes.

\section{Análisis de resultados específicos para: Grupos de Discusión}

Se analizaron los siguientes ítems específicos:

- GP4: Diálogo abierto y participativo

- GP5: Iniciativa para investigar puntos clave

- GP6: Exposición y retroalimentación del docente

De acuerdo con los resultados obtenidos, se observa que la tendencia se centra en valores intermedios y altos de la escala para cada uno de los ítems. También se evidencia que más de la mitad de los encuestados consideran la aplicación de este método en términos regulares o solamente aceptables. Esto se hace más evidente al ser consultado sobre la iniciativa de los estudiantes para investigar puntos clave sobre el tema discutido.

La discusión presupone que los participantes se encuentren con suficientes conocimientos para ser objetivos sobre el tratamiento del tema. Si el método no es altamente valorizado, eso podría estar relacionado a que existe poca orientación previa por parte del docente para que el estudiante realice una preparación adecuada sobre el tema a ser trabajado en cada reunión.

\section{Análisis de resultados específicos para: Seminario}

Se analizaron los siguientes ítems específicos:

- SEM4: Apoyo y orientación del docente

- $\quad$ SEM5: Presentación y análisis de los resultados

- $\quad$ SEM6: Elaboración de conclusiones y documento final 
De acuerdo con los resultados obtenidos, se observa que la tendencia general de respuesta en todos los casos es hacia valores altos de la escala, siendo un poco más leve este comportamiento para el apoyo y orientación del docente. El nivel aceptable de respuestas presenta un mayor porcentaje en todos los ítems. Por otra parte, casi del $60 \%$ de los encuestados valoran de forma aceptable o muy satisfactoria a cada uno de los ítems consultados.

Los resultados también arrojan que aproximadamente una tercera parte de los estudiantes se colocan en una posición neutra en todos los ítems con relación al método y su desarrollo. Eso puede significar que la percepción de esos encuestados es que las directrices en la enseñanza y desarrollo del método no son satisfactorias para su aprendizaje.

\section{Análisis de resultados específicos para: Método de Juego de Roles}

Se analizaron los siguientes ítems específicos:

- $\quad$ MJR4: Análisis de sus propios roles

- $\quad$ MJR5: Aprendizaje significativo y grado de tolerancia alcanzado

- $\quad$ MJR6: Relación del método con el aprendizaje obtenido

la tendencia general de respuesta en todos los casos es hacia valores altos de la escala, siendo un poco menor el nivel de satisfacción cuando se analiza la relación del método con los ítems aprendizaje significativo y grado de tolerancia alcanzado y relación del método con el aprendizaje obtenido.

Adicionalmente, en todos los ítems, más de las dos terceras partes de los encuestados valoran de forma aceptable o muy satisfactoria a cada uno de ellos. El alto involucramiento del estudiante en la aplicación de ese método está reflejado en la elevada valoración obtenida en todos los ítems.

\section{Análisis de resultados específicos para: Aprendizaje Basado en Problemas}

Se analizaron los siguientes ítems específicos:

- $\quad$ ABP4: Presentación del problema y selección del Material de aprendizaje

- ABP5: Rol del docente como tutor

- $\quad$ ABP6: Participación del grupo en la resolución del problema

De acuerdo con los resultados obtenidos, se observa que los niveles de respuesta se concentran en altos y muy altos de la escala para cada uno de los ítems, mientras que la valoración en el nivel aceptable se presenta algo menor para la participación del grupo en la resolución del problema. 
De los resultados obtenidos se despliega que, cuando ese método es utilizado, los estudiantes tienen una valoración alta probablemente porque los aproxima a problemas de la vida real. En el ítem Participación del grupo en la resolución de problema se presenta con una valoración neutral en, aproximadamente, una tercera parte de los encuestados, lo que podría indicar que la enseñanza mediante el trabajo en grupo no aporta mucho para el aprendizaje de esos estudiantes.

\section{Análisis de resultados específicos para: Investigación}

Se analizaron los siguientes ítems específicos:

- INV4: Relación del tema de estudio con resultados de investigación

- INV5: Aplicación del método científico en clase

- INV6: Desarrollo del sentido crítico, análisis, síntesis en los estudiantes

Se observa que los valores para todos los ítems son casi similares y representan la percepción de una tercera parte de los encuestados en lo que se relaciona a la escala más alta. También se evidencia que más del $60 \%$ de los encuestados consideran la aplicación de este método en términos regulares o solamente aceptables en cada uno de los ítems consultados.

Esos resultados pueden ser atribuidos al no dominio, por parte del docente, de la práctica de la investigación para poder enseñar a los estudiantes a aplicar el método científico en las respectivas disciplinas.

\section{Análisis comparativo entre los resultados de las universidades ESPE y UCE}

\section{Preliminares}

Con base en los resultados de la encuesta aplicada a estudiantes del área de Administración, en las Carreras de Administración de Empresas, Contabilidad y Auditoría de la Universidad de las Fuerzas Armadas ESPE y de la Universidad Central, se procedió a realizar un análisis comparativo entre todas estas carreras y las respuestas relacionadas a los distintos métodos de enseñanza contemplados en el instrumento utilizado.

Para esto, en primer lugar, se seleccionaron las respuestas dadas por aquellos estudiantes que respondieron afirmativamente al hecho de que cada método haya sido utilizado en clases. Así se eliminan sesgos de respuesta a favor de determinado método, y se potencializa la discriminación de parte del estudiante, pues cada uno de ellos usó todos los métodos y puede comparar sus bondades y su aplicación en el aula.

Asimismo, se analizaron las respuestas de las preguntas comunes de cada sección. De esta manera, se asegura que cada método pueda ser comparado de forma efectiva, al ser evaluado frente a los mismos ítems y con la misma escala. 
Luego de obtener la base de datos correspondiente, se procedió a realizar el análisis comparativo identificando, en cada caso, la carrera y universidad a la que pertenece cada estudiante encuestado (variable que llamaremos carrera).

El método de análisis estadístico utilizado fue el Análisis de Correspondencias Múltiples $\mathrm{ACM}$, tomando en cuenta que en la base de datos se cuenta únicamente con variables categóricas, y que el objetivo de análisis es reducir las variables e interpretar la información relevante para cada grupo y variable considerada.

El ACM permite construir dimensiones (o ejes) donde se recoge la mayor cantidad de información (variabilidad) y representa gráficamente cada individuo, variable o grupo de interés en función de estos ejes. Esta representación depende del nivel de relación (correlación) del objeto a representar respecto a cada dimensión. Asimismo, permite interpretar cada dimensión en función de aquellas variables más correlacionadas con la misma, y a su vez permite identificar subgrupos, pues aquellos individuos o variables que presenten información similar se correlacionarán de forma parecida en cada dimensión, por lo que su representación gráfica será muy cercana entre sí. Por otra parte, aquellas variables o individuos que tengan menor relación se ubicarán más lejanos, pues sus correlaciones a cada eje (dimensión) también serán distintas.

Para aplicar este análisis, se simplificó la escala de respuestas de la siguiente manera: $1-2$ $=$ Bajo, $3=$ Medio, $4-5=$ Alto. Esto permitió tener el tamaño muestral necesario para cada nivel de la escala (por lo general, hubo pocas respuestas de nivel 1). Esta escala fue recodificada en 3 distintas variables por cada variable original. Por ejemplo, variable CM1, que corresponde a la pregunta "Reconoce que este método fue utilizado de acuerdo con la definición" relacionada al método de Conferencias Magistrales (CM), cuya escala original era de 1 a 5 (variable categórica ordinal), se recodificó en 3 variables: CM_Alto, CM1_Medio, CM1_Bajo, cada una de ellas de carácter nominal, asignándole el valor de CERO o UNO, dependiendo la respuesta del estudiante encuestado.

El ACM se realizó utilizando el software estadístico $\mathrm{R}$, y en especial el paquete "FactoMineR". Detalles sobre este paquete y su utilización para realizar el ACM se puede encontrar en el libro "Exploratory Multivariate Analysis by Example Using R" de Husson, Lê y Pagès (2011).

\section{Análisis generales}

Una vez realizado el ACM, se procede a representar bidimensionalmente la información de cada variable recodificada y de cada individuo. Adicionalmente, se representó la variable "Carrera" donde se indica a que carrera y universidad pertenece cada estudiante. En las Tablas 1 y 2 se presentan las variables más correlacionadas a cada Dimensión o Eje. 
Tabla 1: Variables correlacionadas a la Dimensión 1

\begin{tabular}{ccc}
\hline Variable & $\mathrm{R}^{2}$ & p.value \\
\hline JR1 & 0.640 & 0 \\
SIM1 & 0.584 & 0 \\
JR3 & 0.485 & 0 \\
SIM2 & 0.465 & 0 \\
\hline
\end{tabular}

Fuente: Elaboración propia.

Tabla 2: Variables correlacionadas a la Dimensión 2

\begin{tabular}{ccc}
\hline Variable & $\mathrm{R}^{2}$ & p.value \\
\hline INV1 & 0.299 & 0 \\
SEM3 & 0.245 & 0 \\
ABP1 & 0.242 & 0 \\
\hline
\end{tabular}

Fuente: Elaboración propia.

Como se puede observar, la dimensión 1 está relacionada al uso de los métodos de Juegos de Roles y de Simulación, mientras que la segunda dimensión, implica el uso de Investigación, y Aprendizaje Basado en Problemas. Para conocer el nivel de representatividad de estos ejes, es necesario saber el porcentaje de variabilidad (información) que recogen los mismos. Esto se puede verificar en la Tabla 3 .

Tabla 3: Varianza explicada por las dimensiones 1 a 3

\begin{tabular}{cccc}
\hline Información & Dim.1 & Dim.2 & Dim.3 \\
\hline Varianza explicada & 0.286 & 0.136 & 0.119 \\
\% de varianza & 14.294 & 6.808 & 5.936 \\
\% Acumulado de varianza & 14.294 & 21.103 & 27.039 \\
\hline
\end{tabular}

Fuente: Elaboración propia.

En la tabla anterior, se puede verificar que en las dos dimensiones se puede representar hasta un $21.10 \%$ del total de la información. Este porcentaje se puede considerar bajo, aunque era de esperar, tomando en cuenta que se trata de un estudio exploratorio, y que existen muchas variables (y métodos de enseñanza) que han sido analizados.

Para analizar la información para la variable "Carrera" y como esta se representa en cada dimensión, se construyó la tabla 4, donde se presentan sus coordenadas y el valor de Coseno Cuadrado, que indica el grado de calidad de representación en el plano (a mayor valor, mejor representación. Si este valor es cercano a 1 indica que la representación en el plano es adecuada) 
Tabla 4: Coordenadas y coseno cuadrado de las carreras en el plano

\begin{tabular}{lrrrr}
\hline & \multicolumn{1}{c}{ Dim.1 } & \multicolumn{1}{c}{ Cos $^{2}$} & \multicolumn{1}{c}{ Dim.2 } & \multicolumn{1}{c}{$\operatorname{Cos}^{2}$} \\
\hline COM_ESPE & -0.341 & 0.059 & -0.070 & 0.003 \\
COM_UCE & -0.013 & 0.000 & -0.105 & 0.002 \\
FIN_ESPE & 0.133 & 0.010 & 0.214 & 0.027 \\
FIN_UCE & 0.513 & 0.040 & -0.301 & 0.014 \\
\hline
\end{tabular}

Fuente: Elaboración propia.

Como se puede verificar, la calidad de representación no es tan buena, posiblemente se requieran más dimensiones para representar estas carreras. Esto puede deberse a que existen métodos altamente representativos (como las conferencias magistrales) que no se encuentran representadas en ambas dimensiones). Los resultados anteriores se pueden visualizar en la Figura 1.

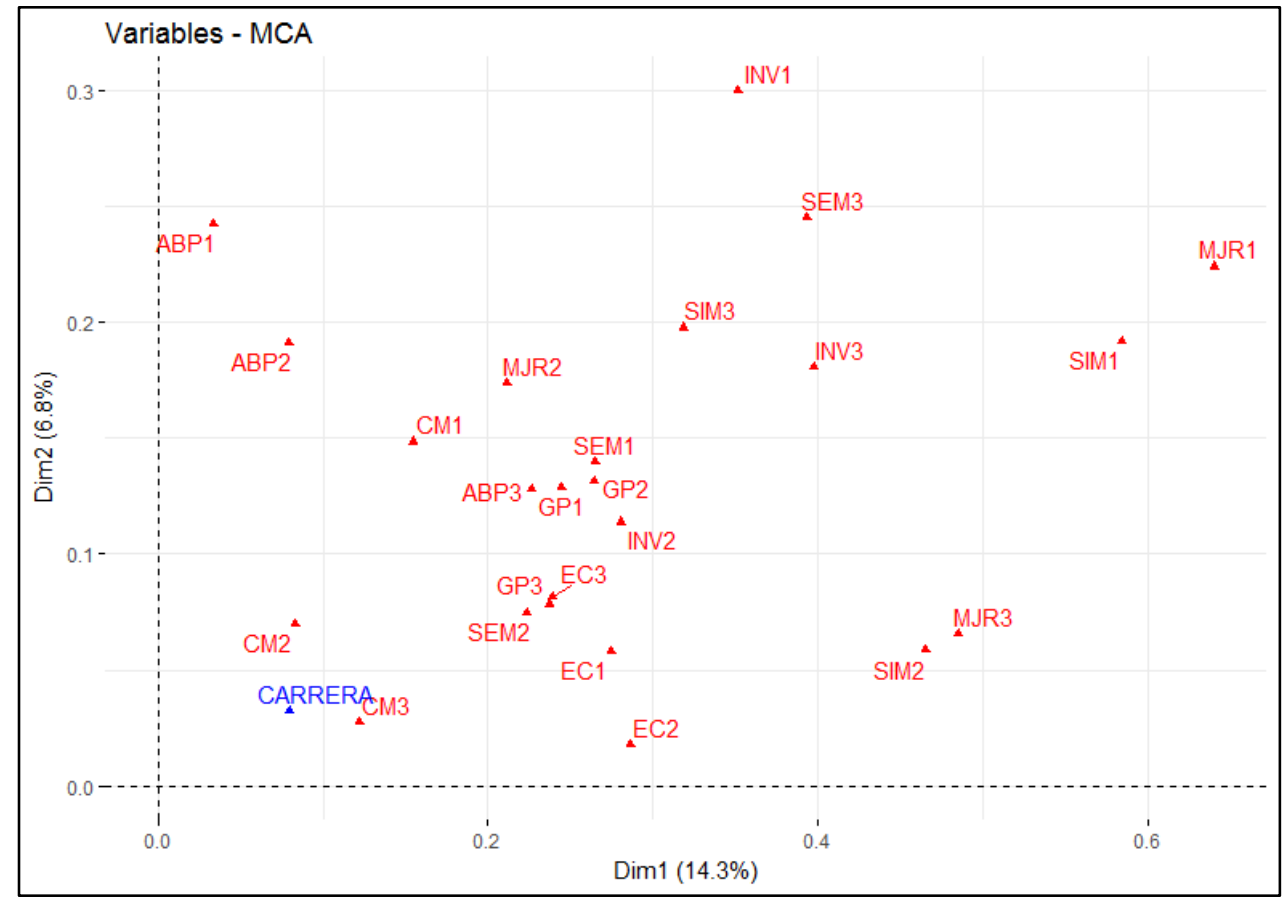

Figura 1: Representación bidimensional de variables

Fuente: Elaboración propia.

En esta figura se observa claramente que las variables relacionadas a Simulación y Juego de Roles tienen mayor correlación con la dimensión 1, mientras que Investigación, Seminarios y Aprendizaje Basado en Problemas están más correlacionados al Eje 2. También se evidencia que las carreras tienen una correlación muy baja en ambos ejes, lo cual se explica a que la mayoría de las respuestas no están representados con estos métodos. 
Para analizar las respuestas dadas por los individuos de cada carrera, y como se relacionan estas con cada eje, se construyó la figura 2. En esta figura se evidencia que la mayoría de los individuos se correlaciona muy poco y de forma negativa con el eje 1 y que su correlación se distribuye de forma casi paralela al eje 2 .

Las elipses de confiabilidad de cada carrera indican que la carrera de Ingeniería Comercial de la ESPE se representa mejor dentro de la nube de puntos, siendo parecida pero más dispersa la información de la misma carrera en la UCE. También se evidencia que la información recogida en la carrera de Ingeniería en Finanza de la UCE es distinta al resto de carreras (se encuentra correlacionada de forma positiva al eje 1), lo cual indica que en esta carrera existe relación con el uso de los métodos de Juegos de Roles y Simulación.

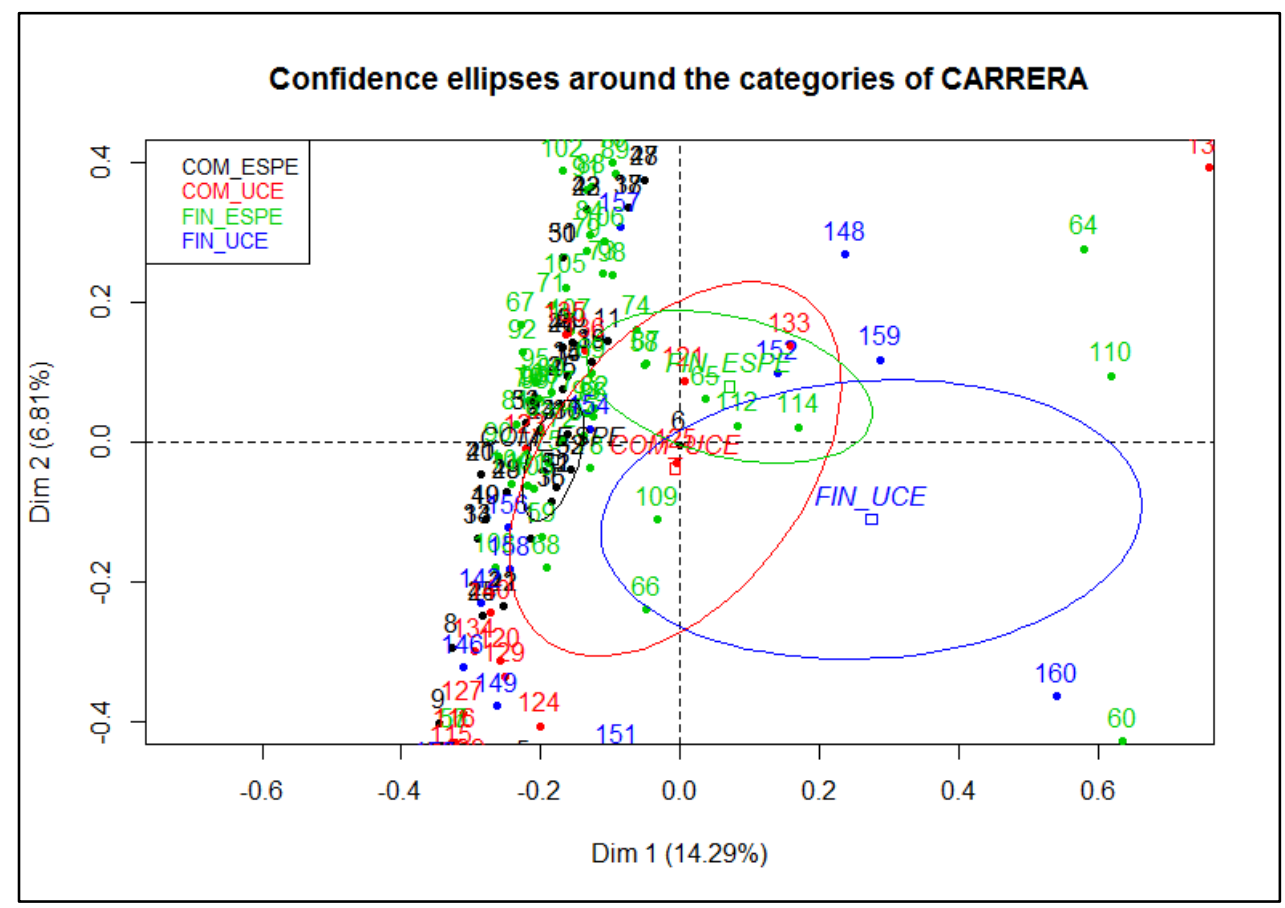

Figura 2: Representación de individuos y elipses de confiabilidad por carrera Fuente: Elaboración propia.

La Figura 3 presenta la representación de cada carrera en función de las variables de escalas de respuesta obtenidas. Aquí se vuelve a evidencias los comportamientos similares entre las carreras de Ing. Comercial de ambas universidades, y que su representación es parecida a la obtenida por los valores altos de la escala (ALTO). La Carrera de Ing. en Finanzas de la ESPE en cambio, tiene mayor relación a valores medios de cada variable, y se observa nuevamente que la Carrera de Finanzas de la UCE se encuentra muy alejada del resto de datos. 


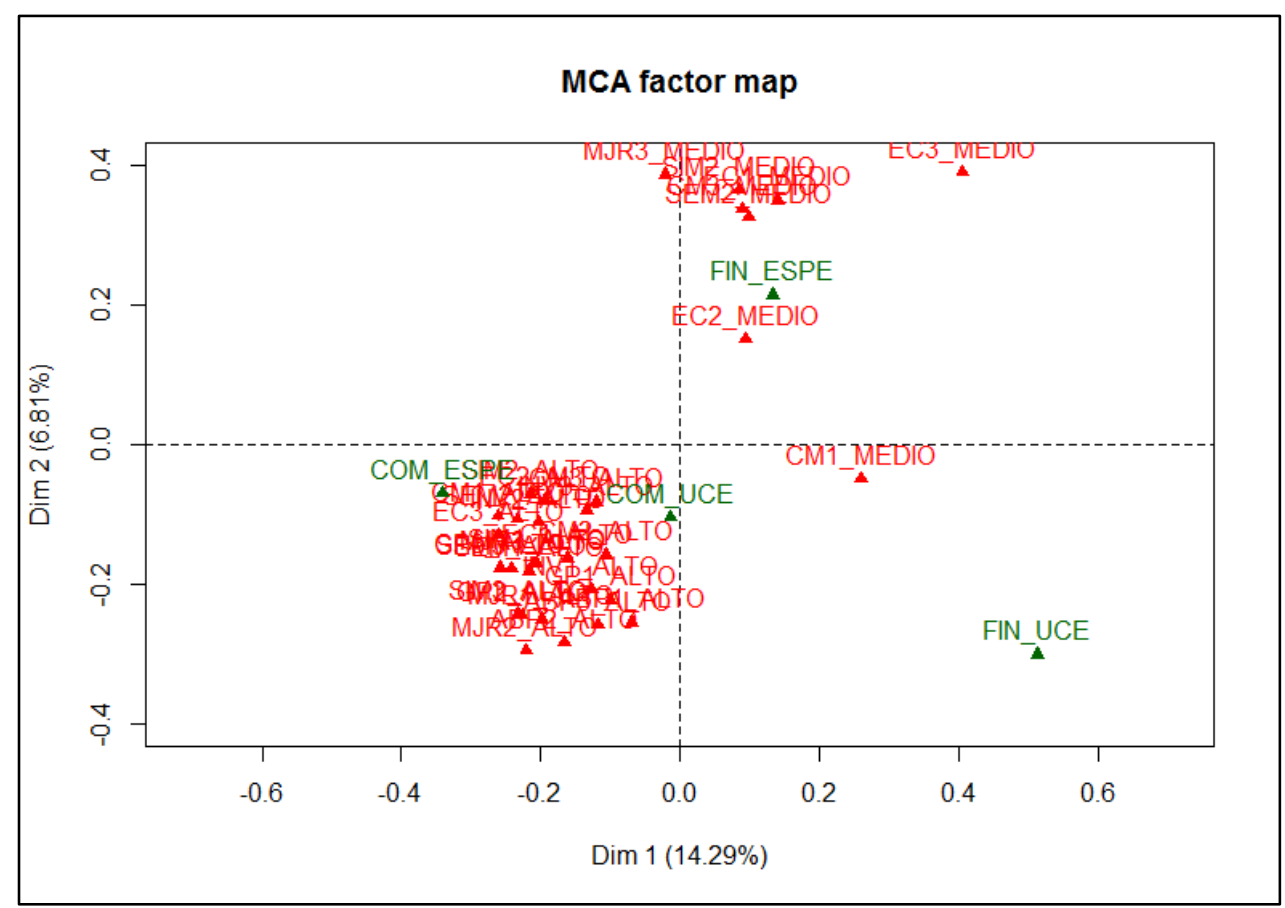

Figura 3: Representación de variables de escalas de respuesta y carreras

Fuente: Elaboración propia.

\section{Conclusiones.}

- De acuerdo con la información obtenida, se observan patrones de respuesta similares entre las carreras de las universidades sujetas a estudio; lo que indica que la metodología de cada universidad no repercute directamente en los métodos aplicados por los docentes, estos últimos aplican sus métodos de acuerdo a la carrera y asignatura independientemente de la institución en la que laboran; al ser carreras técnico - teóricas es aceptable suponer que los docentes apliquen más los métodos de conferencias magistrales y estudios de caso. Pero por parte de los estudiantes no se tienen claridad sobre las características teóricas de cada uno de los métodos para poder compararlas con su aplicación en sala de clase, sin embargo, si son capaces de identificar que los métodos de estudio de caso y la simulación, les han facilitado el aprendizaje de los conocimientos, y que los métodos aprendizaje basado en problemas, grupo de discusión e investigación, lo califican con mayor dificultad para el aprendizaje, esto nos puede demostrar una preparación académica deficiente del docente en la aplicación del método de enseñanza generando una percepción de no aportar para el aprendizaje de los estudiantes.

- Los niveles de representatividad en ambas dimensiones analizadas no es lo suficientemente significativo para inclinarse por ciertas metodologías de enseñanza, pero si se evidencia una clara disposición al poco uso de los métodos correlacionados 
a cada eje (Juego de Roles, Aprendizaje basado en problemas, Seminarios, Simulación).

- Las carreras de ingeniería Comercial en ambas universidades presentan información similar, y se encuentran poco relacionadas a ambos ejes. Ambas carreras también se representan cercanos a valores altos de la escala de respuesta. La carrera de Ingeniería en Finanzas de la ESPE presenta información relaciona a la escala media de respuesta y se correlaciona de forma positiva (aunque con valores bajos) en ambos ejes. Sin embargo, no es factible concluir su comportamiento con algún método en específico. Por otra parte, muestra un comportamiento diferente al resto, y se correlaciona de forma positiva con el eje 1, aunque su nivel de dispersión en las respuestas es bastante alto

\section{Referencias Bibliográficas.}

Aktouf, O. Ensino de administração: por uma pedagogia para a mudança. Organizações \& Sociedade, Salvador, v. 12, n. 35, p. 151-159, 2005.

Álvarez de Zayas C. Hacia una escuela de excelencia. La Habana: Editorial Academia; 2001.

Andrade, Rua Otávio Bernardes de. Gestão de cursos de Administração - metodologias e diretrizes curriculares. São Paulo, SP: Prentice Hall, 2004.

Arreola, R. A. (1995). Developing a comprehensive faculty evaluation system. Bolton, MA: Anker Publishing.

Blaich, C. F., \& Wise, K. S. (2011, January). From gathering to using assessment results: Lessons from the Wabash national study (NILOA Occasional Paper No. 8). Urbana, IL: University of Illinois and Indiana University, National Institute for Learning Outcomes Assessment.

Braskamp, L. A., \& Ory, J. C. (1994). Assessing faculty work: Enhancing individual and instructional performance. San Francisco, CA: Jossey-Bass.

Carreño, Inés (2012). Metodología del Aprendizaje, Editorial Cultura. Madrid.

Casanova Villalba, C. I. (2018). Análisis y mejoramiento de la eficiencia del proceso de emisión de licencias de la agencia nacional de tránsito, Santo Domingo de los Tsáchilas (Master's thesis, PUCE).

Cullen, Carlos A. (1997) Crítica de las razones de educar: temas de filosofía de la educación. Editorial Paidós. Buenos Aires. Argentina. 
Delors, Jacques (Coord.) (1996). Educação: um tesouro a descobrir. Relatório para a UNESCO da comissão internacional sobre educação para o século XXI. Rio Tinto: Edições ASA.

Díaz Barriga F, Hernández G. Estrategias docentes para un aprendizaje significativo. Una interpretación constructivista. México, DF: McGraw-Hill Interamericana Editores, S.A.; 1998.

Herrera Sánchez, M. J. (2018). Mejoramiento de los procesos del área técnica de la unidad administrativa provincial de la Agencia Nacional de Tránsito de Santo Domingo de los Tsáchilas y su impacto en la satisfacción del usuario (Master's thesis, PUCE).

Husson F., Lê S., Pagès J. (2011). Chapman \& Hall/CRC Press, Boca Raton, 240 pp.

Lindberg-Sand, A. and Sonesson, A. (2008) Compulsory higher education teacher training in Sweden: Development of a national standards framework based on the Scholarship of Teaching and Learning. Tertiary Education and Management, 14 (2) 123-39.

Pratt, D. (1997). Reconceptualizing the evaluation of teaching in higher education. Higher Education, 34, 23-44.

Rodríguez, M. (2011). Metodologías docentes en el EEES: de las clases magistrales al portafolio. Tendencias pedagógias, 83-103.

Rosselló, M.R., Didáctica General versus didácticas específicas: un viaje de ida y vuelta, ISSN 2386-7272, Revista Educación y Cultura, 18,133-142 (2005)

Sáez, J. (2018). Estilos de aprendizaje y métodos de enseñanza. Madrid: UNED Publicaciones. 


\section{PARA CITAR EL ARTÍCULO INDEXADO.}

Herrera Enríquez, G., Castillo Páez, S., Zambrano Vera, D., Herrera Sánchez, M. J., \& Casanova Villalba, C. I. (2021). Incidencia de las metodologías de enseñanza en las carreras de ciencias administrativas ofertadas por las universidades públicas del DMQ. Visionario Digital, 5(1), 6-25. https://doi.org/10.33262/visionariodigital.v5i1.1526

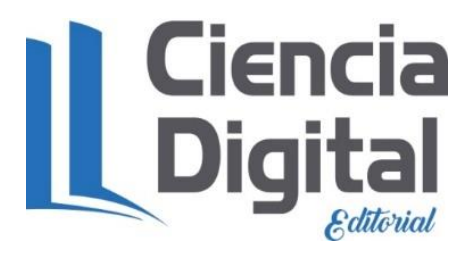

El artículo que se publica es de exclusiva responsabilidad de los autores y no necesariamente reflejan el pensamiento de la Revista Visionario Digital.

El artículo queda en propiedad de la revista y, por tanto, su publicación parcial y/o total en otro medio tiene que ser autorizado por el director de la Revista Visionario Digital.
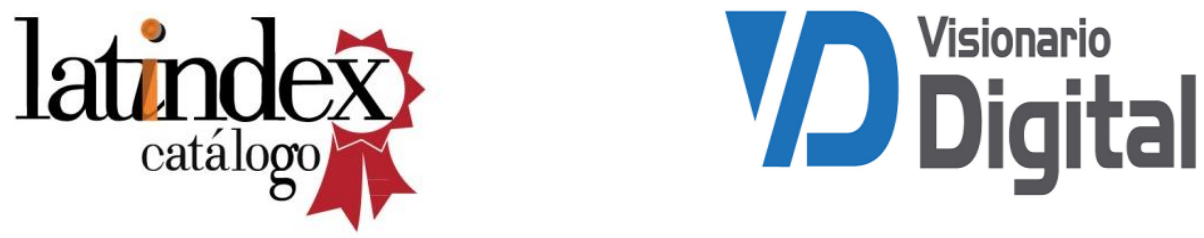